\title{
Magnetic Properties of $\mathrm{Sm}_{2}\left(\mathrm{CO}_{1_{-}} \mathrm{Al}_{x}\right)_{17}$
}

\author{
By Masaaki Hamano*, Seishi Yajima* and Hiromichi Umebayashi**
}

\begin{abstract}
Magnetic properties of $\mathrm{Sm}_{2}\left(\mathrm{Co}_{1-x} \mathrm{Al}_{x}\right)_{17}$ powders with $0 \leqq x \leqq 0.12$ have been investigated at room temperature. The crystal structure of these alloys is of the rhombohedral $\mathrm{Th}_{2} \mathrm{Zn}_{17}$ type. The lattice constants $a$ and $c$ were found to increase monotonically with $x$. The magnetization curves of aligned-powder pellets shows that the decrease of the saturation moment is stronger than that expected for a model of simple dilution of Co moments with non-magnetic Al. In the whole range of $x$ studied these alloys have a magnetically easy $c$-axis. Approximate values of the anisotropy field and the anisotropy constant were estimated from the magnetization curves. The former increases slightly with $x$ and the latter decreases. Curie temperatures of the alloys were determined by the magnetization-temperature curve measured with a magnetic balance.
\end{abstract}

(Received November 29, 1973)

\section{Introduction}

Among the rare-earth(R)-cobalt intermetallic compounds, $\mathrm{RCO}_{5}$ alloys are known as excellent hard magnetic materials because of their high anisotropy constants ${ }^{(1) \sim(4)}$. Some of the $\mathrm{R}_{2} \mathrm{Co}_{17}$ alloys are also promising hard magnetic materials because of their higher magnetic moments and Curie temperatures than $\mathrm{RCo}_{5}$ alloys although they have much lower anisotropy constants ${ }^{(5) \sim(6)}$. Many studies have been made in order to improve their magnetic properties as hard magnetic materials ${ }^{(7) \sim(11)}$.

Previously Zarechnyuk et al. ${ }^{(12)}$ found by the X-ray diffraction studies that there exists a $\mathrm{Ce}_{2} \mathrm{Co}_{15} \mathrm{Al}_{2}$ compound which is isostructural with $\mathrm{Ce}_{2} \mathrm{Co}_{17}$. If this result can be extended to other $\mathrm{R}_{2} \mathrm{Co}_{17}$ compounds, it is expected that Co atoms may be replaced by non-magnetic $\mathrm{Al}$ atoms, resulting in a systematic change in magnetic properties of $\mathrm{R}_{2} \mathrm{Co}_{17}$.

In this work we selected $\mathrm{Sm}$ atoms as $\mathrm{R}$ and investigated the change in magnetic properties with $\mathrm{Al}$ content in the $\mathrm{Sm}_{2}\left(\mathrm{Co}_{1-x} \mathrm{Al}_{x}\right)_{17}$ compounds.

\section{Experimental}

The alloys were prepared by arc melting of the starting materials in an argon atmosphere. The buttons weighing approximately $12 \mathrm{~g}$ were melted carefully to ensure their homogeneity. Purities of starting Sm, Co, and Al metals were $99.8 \%, 99.9 \%$, and $99.99 \%$, respectively. Vacuum annealing of the buttons was performed in sintered $\mathrm{Al}_{2} \mathrm{O}_{3}$ boats at $1100^{\circ} \mathrm{C}$ for $24 \mathrm{hr}$. Phase analyses of the alloys were carried out by $\mathrm{X}$-ray diffraction. The composition of the singlephase compounds thus obtained was determined by chemical analysis. Lattice parameters were measured with a Debye-Sherrer camera. Magnetically oriented pellets were prepared by solidifing the powders of the alloys with wax in a magnetic field of about $7 \mathrm{kOe}$.

* The Oarai Branch, The Research Institute for Iron, Steel and Other Metals, Tohoku University, Oarai, Ibaraki 311-13, Japan.

** Metropolitan College of Technology, Tokyo, Hino, Tokyo 191, Japan.

Trans. JIM
The crystallographic easy direction of magnetization was determined by a comparison of X-ray diffraction patterns taken for both faces of the pellets parallel $(\|)$ and perpendicular $(\perp)$ to the magnetic field applied during solidification. Magnetization curves were measured on the easy and hard directions of the oriented pellets at room temperature using an inducting magnetometer under a maximum field of 13.3 kOe. Curie temperatures of the alloys were measured with a magnetic balance.

\section{Results and Discussion}

According to the X-ray diffraction experiments the alloys with the single phase of the rhombohedral $\mathrm{Th}_{2} \mathrm{Zn}_{17}$ type structure have been obtained in the range of $0.12 \geqq x \geqq 0$ in the $\mathrm{Sm}_{2}\left(\mathrm{Co}_{1-x} \mathrm{Al}_{x}\right)_{17}$ system. Lattice parameters, $a$ and $c$ in the hexagonal expression, are shown in Fig. 1. The values of $\mathrm{Al}$ content shown in the figure are nominal, which have been found to be essentially identical with real values determined by the chemical analysis. Both $a$ and $c$ increase monotonically with increasing $\mathrm{Al}$ contents. This increment is reasonable because Al has a larger atomic radius than Co replaced.

The crystallographic axes of the easy magnetization are found to be the $c$-axis for all the samples studied. It is known that the magnetic anisotropy of some

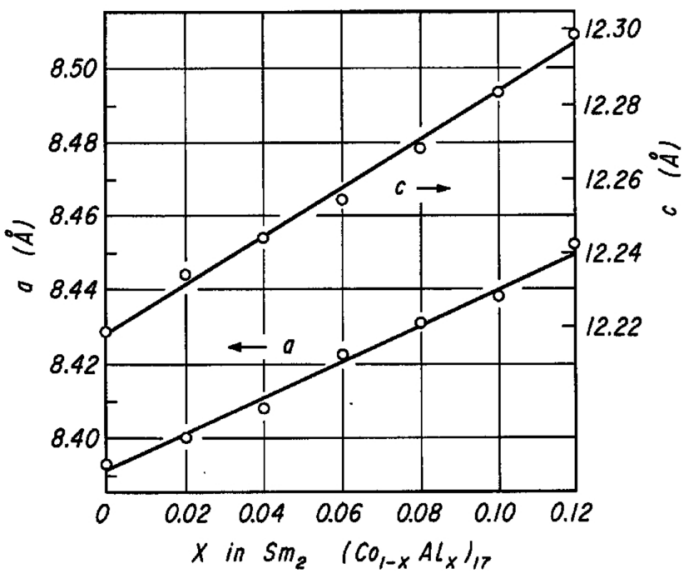

Fig. 1 The variation of lattice parameters with $\mathrm{Al}$ content.

1974 Vol. 15 


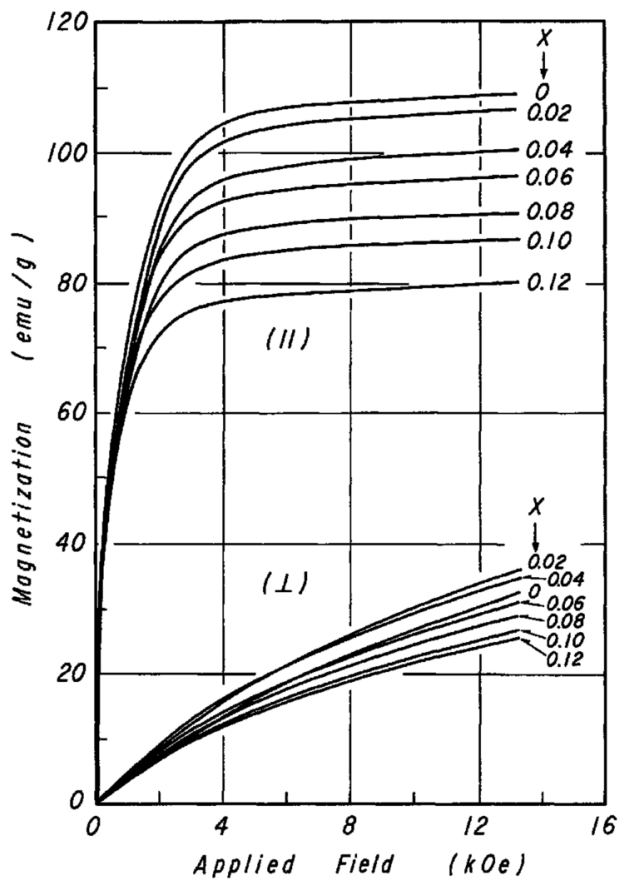

Fig. 2 Magnetization curves of aligned-powder pellets of $\mathrm{Sm}_{2}\left(\mathrm{Co}_{1-x} \mathrm{Al}_{x}\right)_{17}$ compounds at room temperature. The measured directions are parallel (II) and perpendicular $(\perp)$ to the magnetic field applied during solidification.

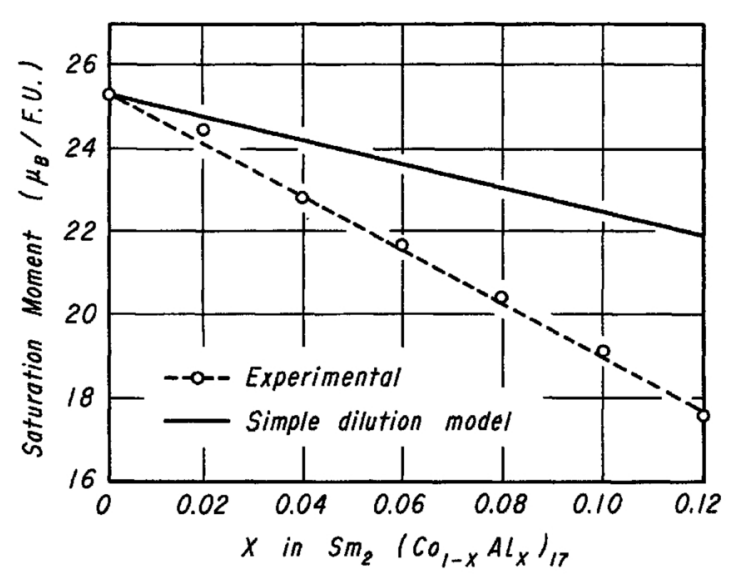

Fig. 3 The variation of saturation moments at room temperature as a function of $\mathrm{Al}$ content.

$\mathrm{R}_{2} \mathrm{Co}_{17}$ compounds change the sign sensitively according to the addition of the third component or to the degree of stoichiometry ${ }^{(7) \sim(10)}$. In case of the present $\mathrm{Sm}_{2}\left(\mathrm{Co}_{1-x} \mathrm{Al}_{x}\right)_{17}$ system, the magnetic anisotropy constant remains positive and no change in the sign has been observed. Magnetization vs applied field curves for both easy and hard directions of the oriented pellets are shown in Fig. 2. The saturation magnetization $\left(I_{s}\right)$ decreases with increasing $\mathrm{Al}$ content. Figure 3 shows the plots of the saturation moment per formula unit ( $\mu_{B} /$ F.U.) vs $x$. The solid line in the figure is calculated based upon a simple dilution model, where the magnetic moments of Co atoms are assumed to be $1.61 \mu_{B}$ irrespective of the value of $x$ and Co atoms are simply replaced by the nonmagnetic $\mathrm{Al}$ atoms. The decrease of the saturation moment is seen to be stronger than that expected for the simple dilution model. A similar phenomenon was reported by Schaller et al. ${ }^{(13)}$ in the $\mathrm{Y}_{2} \mathrm{Co}_{17-x} \mathrm{~T}_{x}$ $(\mathrm{T}=\mathrm{Mn}, \mathrm{Ru})$ system. In their paper, the rapid decrease of the Co moment was attributed to two possible situations: (1) the coupling between Co and $\mathrm{T}$ is antiferromagnetic, and (2) outer electrons from $T$ fill up $3 \mathrm{~d}$ holes of Co resulting in the reduction of Co moments. In the present study, the latter explanation may be applicable.

Approximate values of anisotropy fields $\left(H_{a}\right)$ were estimated by extrapolating the linear portions of the magnetization curves for both $(\|)$ and $(\perp)$ directions. The straight line portions in the curves for $(\perp)$ direction over a range of applied fields from 10.0 to 13.3 $\mathrm{kOe}$ were shifted to pass through the origin on the graph for the purpose of making a rough correction of the imcomplete alignment of the crystallites in the pellets. Using these anisotropy fields, anisotropy constants $(K)$ were calculated from the following equation:

$$
K=I_{s} \cdot H_{a} / 2 \text {. }
$$

It is to be noted that the above discussion is rather immature for the following two reasons and that the results should be understood to give only roughly estimated values of the anisotropy constants; (1) the easy axes of the crystallite in the sample is not completely aligned, and (2) higher order anisotropy constants are neglected. The values of saturation magnetizations, anisotropy fields and anisotropy constants thus obtained are shown in Table 1. The anisotropy field seems to increase slightly with increasing $\mathrm{Al}$ content, but the anisotropy constant decreases slowly owing to a rapid decrease of the magnetic moment. It can be said that the addition of non-magnetic $\mathrm{Al}$ does not have any vital effects on the magnetic anisotropy of the $\mathrm{Sm}_{2} \mathrm{Co}_{17}$ compound. The Curie temperature was found to decrease monotonically

Table 1 Some magnetic properties of aligned-powder pellets of $\operatorname{Sm}_{2}\left(\mathrm{Co}_{1-x} \mathrm{Al}_{x}\right)_{17}$ compounds at room temperature.

\begin{tabular}{|c|c|c|c|c|c|}
\hline \multirow{2}{*}{ in $\stackrel{x}{\mathrm{Sm}_{2}\left(\mathrm{Co}_{1-x} \mathrm{Al}_{x}\right)_{17}}$} & \multicolumn{2}{|c|}{ Saturation magnetization } & \multirow{2}{*}{$\begin{array}{l}\text { X-ray density } \\
\rho_{x}\left(\mathrm{~g} / \mathrm{cm}^{3}\right)\end{array}$} & \multirow{2}{*}{$\begin{array}{c}\text { Anisotropy field } \\
H_{a}(\mathrm{kOe})\end{array}$} & \multirow{2}{*}{$\begin{array}{c}\text { Anisotropy constant } \\
K\left(10^{7} \mathrm{erg} / \mathrm{cc}\right)\end{array}$} \\
\hline & $I_{s}(\mathrm{emu} / \mathrm{g})$ & $4 \pi \mathrm{M}_{s}(\mathrm{kG})$ & & & \\
\hline 0 & 109 & 11.9 & 8.70 & 72 & 3.4 \\
\hline 0.02 & 107 & 11.5 & 8.60 & 75 & 3.4 \\
\hline 0.04 & 100 & 10.7 & 8.51 & 79 & 3.3 \\
\hline 0.06 & 96.7 & 10.2 & 8.40 & 80 & 3.2 \\
\hline 0.08 & 91.0 & 9.49 & 8.30 & 81 & 3.0 \\
\hline 0.10 & 86.7 & 8.94 & 8.21 & 78 & 2.8 \\
\hline 0.12 & 80.1 & 8.15 & 8.10 & 84 & 2.7 \\
\hline
\end{tabular}




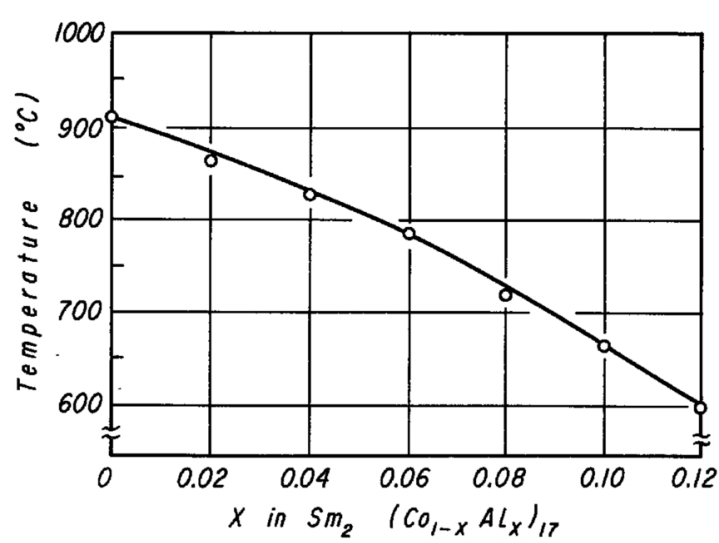

Fig. 4 Curie temperatures of $\mathrm{Sm}_{2}\left(\mathrm{Co}_{1-x} \mathrm{Al}_{x}\right)_{17}$ compounds.

with increasing Al content, as shown in Fig. 4.

Aluminum atoms were expected to replace cobalt atoms preferentially at a particular crystallographic site as is typically realized in the $\mathrm{Ce}_{2} \mathrm{Co}_{15} \mathrm{Al}_{2}$ compound. The efforts to determine the site of $\mathrm{Al}$ atoms in a $\mathrm{Sm}_{2}\left(\mathrm{Co}_{0.88} \mathrm{Al}_{0.12}\right)_{17}$ crystal by powder $\mathrm{X}$-ray diffraction have not been successful, because the differences of the calculated intensities among the various models of atomic distribution were extremely small and were exceeded by the experimental errors.

\section{REFERENCES}

(1) G. Hoffer and K. J. Strnat: J. Appl. Phys., 38 (1967), 1377.

(2) W. A. J. J. Velge and K. H. J. Buschow: J. Appl. Phys., 39 (1968), 1717.

(3) K. H. J. Buschow and W. A. J. J. Velge: Z. angew. Phys., 26 (1969), 157.

(4) D. L. Martin and M. G. Benz: Cobalt, 50 (1971), 11.

(5) K. J. Strnat: Cobalt, 36 (1967), 133.

(6) D. Lemaire, Runé Pauthenet and J. Schweizer: IEEE Trans. Magn., MAG-6 (1970), 153.

(7) S. Yajima, M. Hamano and H. Umebayashi: J. Phys. Soc. Japan, 32 (1972), 861.

(8) M. Hamano, S. Yajima and H. Umebayashj: IEEE Trans. Magn., MAG-8 (1972), 518.

(9) K. J. Strnat: IEEE Trans. Magn., MAG-8 (1972), 511.

(10) G. Hilscher, H. Kirchmayr, W. Steiner and G. Wiesinger: Proc. 10th Rare Earth Res. Conf., Carefree, Arizona, USA, (1973), p. 301.

(11) H. Mildrum, J. Tront, M. Hartings and K. J. Strnat: Proc. 10th Rare Earth Res. Conf., Carefree, Arizona, USA, (1973), p. 476.

(12) O. S. Zarechnyuk and P. I. Kripyakevich: Sov. Phys. Cryst., 7 (1963), 436.

(13) H. J. Schaller, R. S. Craig and W. E. Wallace: J. Appl. Phys., 43 (1972), 3161. 